CARPATHIAN J. MATH.

Volume 38 (2022), No. 1,

Pages 21 - 34
Online version at https : //www. carpathian. cunbm. utcluj. ro/

Print Edition: ISSN 1584 - 2851; Online Edition: ISSN 1843 - 4401

DOI: https://doi.org/10.37193/CJM.2022.01.03

Dedicated to the memory of Academician Mitrofan M. Choban (1942-2021)

\title{
Distance-transitive strongly regular graphs
}

\author{
MONTHER RASHED ALFuraidan
}

\begin{abstract}
We present a complete description of strongly regular graphs admitting a distance-transitive group of automorphisms. Parts of the list have already appeared in the literature; however, this is the first time that the complete list appears in one place. The description is complemented, where possible, with the discussion of the corresponding distance-transitive groups and some further properties of the graphs. We also point out an open problem.
\end{abstract}

\section{INTRODUCTION}

A distance-transitive graph $G$ is one about to which the automorphism group acts transitively on every ordered pairs of vertices at same distance. We only need to consider connected graphs since any connected component of a disconnected distance-transitive graph is itself distance-transitive. In fact, all the connected components, in such a graph, are isomorphic. As a distance-transitive graph is vertex-transitive, it is natural to ask whether the automorphism group of such a graph is vertex primitive or imprimitive. The rank 3 graphs are the distance-transitive graphs of diameter two. Such graphs studied carefully by Donald G. Higman in his breakthrough article [18], also see [10].

An enormous work has done into the classification of all finite distance-transitive graphs. The classification obviously breakdowns into two portions-primitive and imprimitive. The main portion of the classification is the primitive finite distance-transitive graphs, which appears to be completely finished. For the imprimitive graphs, Derek Smith [29] proved that the possibilities for nontrivial blocks of imprimitivity are strictly limited. In this case, we can reduce the given imprimitive distance-transitive graph to a primitive distance-transitive graph. Brouwer and Van Bon [7], Jurišić [24, 25], and Hemmeter [16, 17] worked on the reverse of Smith's theorem, characterizing any associated imprimitive distance-transitive graphs for most of the known primitive distance-transitive graphs

In [6] J. I. Hall and the current author presented a detailed version of Smith's theorem which implies the primitive distance-transitive graph of diameter bigger than one and valency bigger than two is the only core for any unknown imprimitive distance-transitive graph. In [5] J. I. Hall and the current author nearly closed the classification of all imprimitive distance-transitive graphs with primitive core of diameter bigger than two by using the results of Brouwer and Van Bon [7] and Hemmeter [16, 17]. The missing class of graphs is recently closed in [4].

In [2] and [3] the current author classify all imprimitive distance-transitive graphs with primitive core of diameter two.

We are only considering finite graphs and groups.

Received: 23.02.2021. In revised form: 28.10.2021. Accepted: 04.11.2021

2010 Mathematics Subject Classification. 05E30, 5C25.

Key words and phrases. strongly regular graphs, distance-transitive group. 


\section{PRELIMINARIES}

Let $G$ be a connected graph of diameter $d$. Denote by $G_{i}(x)$ the set of vertices of $G$ at distance $i$ from the vertex $x$ in $G$.

For $y \in G_{i}(x)$ set

$$
\begin{aligned}
a_{i}^{x, y} & =\left|G_{i}(x) \cap G_{1}(y)\right| ; \\
b_{i}^{x, y} & =\left|G_{i+1}(x) \cap G_{1}(y)\right| ; \\
c_{i}^{x, y} & =\left|G_{i-1}(x) \cap G_{1}(y)\right| .
\end{aligned}
$$

The graph $G$ is a distance-regular graph if, for all $0 \leq i \leq d$, each of the parameters $a_{i}^{x, y}$, $b_{i}^{x, y}$, and $c_{i}^{x, y}$ depends not on the choice of $\mathrm{x}$ and $\mathrm{y}$ but only on $i$. In that case we will write

$$
\begin{aligned}
a_{i}^{x, y} & =a_{i}=a_{i}(G) ; \\
b_{i}^{x, y} & =b_{i}=b_{i}(G) ; \\
c_{i}^{x, y} & =c_{i}=c_{i}(G) .
\end{aligned}
$$

Often one writes $\lambda=a_{1}$ and $\mu=c_{2}$. Trivially $a_{0}=c_{0}=b_{d}=0$ and $c_{1}=1$. We let $k_{i}=\left|G_{i}(x)\right|$ (a constant) so that $k_{0}=1$. Set $k=k_{1}(G)=b_{0}$, the valency of $G$. Then $k=a_{i}+b_{i}+c_{i}$ for $0 \leq i \leq d$. The sequence

$$
i(G)=\left\{b_{0}, b_{1}, \ldots, b_{d-1} ; c_{1}, c_{2}, \ldots, c_{d}\right\}
$$

is called the intersection array of $G$ and contains all the necessary parameter information (see Proposition 4.1). Clearly a distance-transitive graph is distance-regular, but the converse is in general false.

The distance regular graph of diameter two with intersection array

$$
\{k, k-\lambda-1 ; 1, \mu\}=\left\{b_{0}, b_{1} ; c_{1}, c_{2}\right\}
$$

is the connected strongly regular graph $G=(n, k, \lambda, \mu)$. The complement of a strongly regular graph with parameters $(n, k, \lambda, \mu)$ is strongly regular with parameters $(n, n-k-$ $1, n-2 k+\mu-2, n-2 k+\lambda)$.

A Latin square of order $n$ is an $n \times n$ array filled with $n$ different entries, each occurring exactly once in each row and exactly once in each column.

An orthogonal array $O A(n, m)$ with parameters $n$ and $m$ is a $n \times m^{2}$ array whose entries are chosen from a set $X$ with $m$ points such that the $m^{2}$ ordered pairs defined by any two rows of the array are all distinct. A Latin square is therefore an $O A(3, m)$.

Given $O A(n, m)$, consider the graph $G$ with vertex set the $m^{2}$ columns of length $n$ and two vertices are connected by an edge if they have the same entries in one coordinate position. Then $G$ is strongly regular with parameters

$$
\left(m^{2},(m-1) n, m-2+(n-1)(n-2), n(n-1)\right) .
$$

This graph is called a Latin square graph since an orthogonal array $O A(n, m)$ is equivalent to $n-2$ mutually orthogonal Latin squares of order $m$. A strongly regular graph with parameters

$$
\left(m^{2},(m+1) n,-m-2+(n+1)(n+2), n(n+1)\right)
$$

is known as a negative Latin square graph.

\section{THE FiRst STEP - O'NAN-SCOT THEOREM}

The first analysis of finite primitive distance-transitive graphs using $\mathrm{O}^{\prime} \mathrm{Nan}-\mathrm{Scot}$ theorem was given by Praeger, Saxl and Yokoyama [28]. Their result is the first step toward the classification of finite primitive DTG's. 
Theorem 3.1. Let $A$ act distance transitively on a primitive distance-transitive graph $G$. Then one of the following holds.

- $G$ is a Hamming graph $H(n, q)$ or the complement of a Hamming graph $H(2, q)$ and $A$ is a wreath product. (In this case, the graph $G$ is well known but the possibilities for the group $A$ are not completely determined).

- A has an elementary abelian normal subgroup which is regular on $V(G)$. (This case is referred to as the affine type).

- A has a simple socle. That is, there is a simple nonabelian normal subgroup $N$ of $A$ such that A canonically embeds in $A u t(N)$ (that is, the centralizer $C_{G}(N)$ of $N$ in $A$ is trivial). (This case is referred to as the simple socle or almost simple type).

As a summary, we have the following tree.

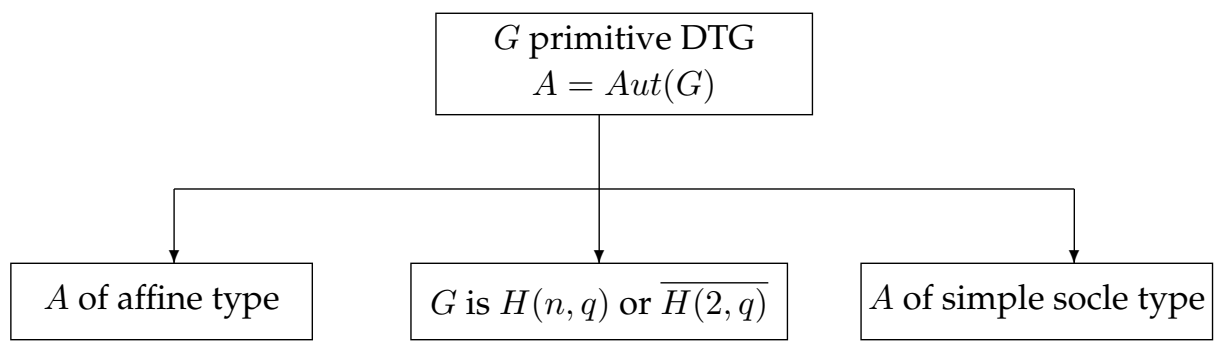

FIGURE 1. primitive main tree

\section{PRIMitive DTGS OF DiAMETER tWo OF AFFINE TYPE}

In this section, we discuss the classification of the primitive affine DTGs of diameter two.

The main example of graphs admitting distance-transitive action of affine type is the Hamming graph $H(n, q)$. The bilinear forms graph $H_{q}(n, m)$ gives another classical example of an affine DTG. Further examples can be constructed as follows.

The Hermitian forms graphs $\operatorname{Her}(n, q)$ (where $n, q>1$ ) have as vertices the $n \times n$ Hermitian matrices over $\mathbb{F}_{q}$ (where $q=p^{2}, p$ a prime power) with two matrices joined by an edge if and only if their difference has rank 1. (see [9, sec. 9.5])

The alternating forms graphs $\operatorname{Alt}(n, q)$ (where $n, q>1$ ) have as vertices the $n \times n$ alternating matrices over $\mathbb{F}_{q}$, that is, all $n \times n$ matrices $\left(a_{i j}\right)_{n \times n}$ with $a_{i j}=-a_{j i}$, for $1 \leq i, j \leq n$, and $a_{i i}=0$ for all $i$, with two matrices joined by an edge if and only if their difference has rank 1 . (see [9, sec. 9.5])

Now let us consider the general classification scheme for the primitive DTGs of diameter two of affine type. Let $G$ be an affine DTG of diameter two with $A u t(G)=A$. Then $V(G)$ can be identified with a vector space $V$ over the field $\mathbb{F}_{s}$ of order $s$ for some power $s=r^{b}$ of a prime $r$, maximal with respect to $A_{0} \leq G L(V)$, where $A_{0}$ is the stabilizer in $A$ of $0 \in V$. And $A$ acts as a primitive affine rank 3 permutation group on $V$. The following theorem due to Liebeck [21] gives a complete classification of such actions. Not all of them though lead to a distance-transitive graph. If each orbit of $A$ is left invariant under multiplication by -1 , then it is distance-transitive. 
Theorem 4.2. Let $A$ be a finite primitive affine permutation group of rank 3 and of degree $n=p^{d}$, with socle $V$, where $V \cong\left(Z_{p}\right)^{d}$ for some prime $p$, and let $A_{0}$ be the stabilizer of the zero vector in $V$. Then $A_{0}$ belongs to one of the following classes (and conversely, each of the possibilities listed below does give rise to a rank 3 affine group).

(A) Infinite classes. These are

(1) $A_{0} \leq \Gamma L_{1}\left(p^{d}\right)$ : all possibilities for $A_{0}$ are determined in [13, sec 3];

(2) $A_{0}$ imprimitive: $A_{0}$ stabilizes a pair $\left\{V_{1}, V_{2}\right\}$ of subspaces of $V$, where $V=V_{1} \oplus V_{2}$ and $\operatorname{dim} V_{1}=\operatorname{dim} V_{2}$; moreover, $\left(A_{0}\right)_{V_{i}}$ is transitive on $V_{i} \backslash 0$ for $i=1,2$ (and hence $A_{0}$ is determined by Hering's Theorem);

(3) tensor product case: for some $a, q$ with $q^{a}=p^{d}$, consider $V$ as a vector space $V_{a}(q)$ of dimension a over $G F(q)$; then $A_{0}$ stabilizes a decomposition of $V_{a}(q)$ as a tensor product $V_{1} \otimes V_{2}$ where $\operatorname{dim}_{G F(q)} V_{1}=2$; moreover, $A_{0}^{V_{2}} \triangleright S L\left(V_{2}\right)$, or $A_{0}^{V_{2}}=A_{7}<S L_{4}(2)$ (and $p=q=2, d=a=8)$, or $\operatorname{dim}_{G F(q)} V_{2} \leq 3$;

(4) $A_{0} \triangleright S L_{a}(q)$ and $p^{d}=q^{2 a}$;

(5) $A_{0} \triangleright S L_{2}(q)$ and $p^{d}=q^{6}$;

(6) $A_{0} \triangleright S U_{a}(q)$ and $p^{d}=q^{2 a}$;

(7) $A_{0} \triangleright \Omega_{2 a}^{ \pm}(q)$ and $p^{d}=q^{2 a}$ (and if $q$ is odd, $A_{0}$ contains an automorphism interchanging the two orbits of $\Omega_{2 a}^{ \pm}(q)$ on non-singular 1-spaces);

(8) $A_{0} \triangleright S L_{5}(q)$ and $p^{d}=q^{10}$ (from the action of $S L_{5}(q)$ on the skew square $\bigwedge^{2}\left(V_{5}(q)\right)$ );

(9) $A_{0} / Z\left(A_{0}\right) \triangleright \Omega_{7}(q) \cdot Z_{(2, q-1)}$ and $p^{d}=q^{8}$ (from the action of $B_{3}(q)$ on a spin module);

(10) $A_{0} / Z\left(A_{0}\right) \triangleright P \Omega_{10}^{+}(q)$ and $p^{d}=q^{16}$ (from the action of $D_{5}(q)$ on a spin module);

(11) $A_{0} \triangleright S z(q)$ and $p^{d}=q^{4}$ ( from the embedding $S z(q)<S p_{4}(q)$ ).

(B) Extraspecial classes. Here $A_{0} \leq N_{G L_{d}(p)}(R)$ where $R$ is an r-group, irreducible on $V$. Either $r=3$ and $R \cong 3^{1+2}$ (extraspecial of order 27 ) or $r=2$ and $|R / Z(R)|=2^{2 m}$ with $m=1$ or 2 . If $r=2$ then either $|Z(R)|=2$ and $R$ is one of the two extraspecial groups $R_{1}^{m}, R_{2}^{m}$ of order $2^{1+2 m}$, or $|Z(R)|=4$, when we write $R=R_{3}^{m}$. The possibilities are listed in Table 1 .

(C) Exceptional classes. Here the socle $L$ of $A_{0} / Z\left(A_{0}\right)$ is simple, and the possibilities are given in Table 2. 
TABLE 2. (C) Extraspecial Classes

TABLE 1. (B) Extraspecial Classes

\begin{tabular}{|c|c|c|}
\hline$r$ & $p^{d}$ & $R$ \\
\hline 3 & $2^{6}$ & $3^{1+2}$ \\
\hline & $3^{4}$ & \\
& $7^{2}$ & \\
& $13^{2}$ & \\
& $17^{2}$ & \\
2 & $19^{2}$ & $R_{1}^{1}$ or $R_{2}^{1}$ \\
& $23^{2}$ & (i.e $D_{8}$ or $\left.Q_{8}\right)$ \\
& $3^{6}$ & \\
& $29^{2}$ & \\
& $31^{2}$ & \\
& $47^{2}$ & \\
\hline & $3^{4}$ & $R_{1}^{2}$ \\
& $3^{4}$ & $R_{2}^{2}$ \\
2 & $5^{4}$ & $R_{2}^{2}$ \\
& $5^{4}$ & $R_{3}^{2}$ \\
& $7^{4}$ & $R_{2}^{2}$ \\
& $3^{8}$ & $R_{2}^{3}$ \\
\hline
\end{tabular}

\begin{tabular}{|c|c|c|}
\hline$L$ & $p^{d}$ & embedding of $L$ \\
\hline & $3^{4}$ & \\
& $31^{2}$ & \\
& $41^{2}$ & \\
$A_{5}$ & $7^{4}$ & $A_{5}<P S L_{2}\left(p^{d / 2}\right)$ \\
& $71^{2}$ & \\
& $79^{2}$ & \\
& $89^{2}$ & \\
\hline$A_{6}$ & $2^{6}$ & $A_{6}<P S L_{3}(4)$ \\
& $5^{4}$ & $A_{6}<P S p_{4}(5)$ \\
\hline$A_{7}$ & $2^{8}$ & $A_{7}<P S L_{4}(4)$ \\
& $7^{4}$ & $A_{7}<P S p_{4}(7)$ \\
\hline$A_{9}$ & $2^{8}$ & $A_{9}<\Omega_{8}^{+}(2)$ \\
\hline$A_{10}$ & $2^{8}$ & $A_{10}<S p_{8}(2)$ \\
\hline$P S L_{2}(17)$ & $2^{8}$ & $P S L_{2}(17)<S p_{8}(2)$ \\
\hline$P S L_{3}(4)$ & $3^{6}$ & $P S L_{3}(4)<P \Omega_{6}^{-}(3)$ \\
\hline$P S U_{4}(2)$ & $7^{4}$ & $P S U_{4}(2)<P S L_{4}(7)$ \\
\hline$M_{11}$ & $3^{5}$ & $M_{11}<P S L_{5}(3)$ \\
\hline$M_{24}$ & $2^{11}$ & $M_{24}<P S L_{11}(2)$ \\
\hline Suz & $3^{12}$ & $S u z<P S p_{12}(3)$ \\
\hline$G_{2}(4)$ & $3^{12}$ & $G_{2}(4)<S u z<P S p_{12}(3)$ \\
\hline$J_{2}$ & $2^{12}$ & $J_{2}<G_{2}(4)<S p_{6}(4)$ \\
& $5^{6}$ & $J_{2}<P S p_{6}(5)$ \\
\hline & \multicolumn{2}{|c}{} \\
\hline
\end{tabular}

Hence as a summary we do have the following tables which gives us all the rank three graphs corresponding to these actions.

TABle 3. $\operatorname{Aut}(G)$ in class $A$ of theorem

\begin{tabular}{|c|c|c|c|}
\hline Type of $A=A u t(G)$ & $n$ & $k$ & $\lambda$ \\
& & $l$ & $\mu$ \\
\hline (A2): $A_{0}$ imprimitive & $p^{2 m}$ & $2\left(p^{m}-1\right)$ & $p^{m}-2$ \\
& & $\left(p^{m}-1\right)^{2}$ & 2 \\
\hline (A3): tensor product & $q^{2 m}$ & $(q+1)\left(q^{m}-1\right)$ & $q^{m}+q^{2}-q-2$ \\
& & $q\left(q^{m}-1\right)\left(q^{m-1}-1\right)$ & $q(q+1)$ \\
\hline (A4): $A_{0} \triangleright S L_{a}(q)$ & $q^{2 a}$ & $(q+1)\left(q^{a}-1\right)$ & $q^{a}+q^{2}-q-2$ \\
& & $q\left(q^{a}-1\right)\left(q^{a-1}-1\right)$ & $q(q+1)$ \\
\hline (A5): $A_{0} \triangleright S L_{2}(q)$ & $q^{6}$ & $(q+1)\left(q^{3}-1\right)$ & $q^{3}+q^{2}-q-2$ \\
& & $q\left(q^{3}-1\right)\left(q^{2}-1\right)$ & $q(q+1)$ \\
\hline (A6): $A_{0} \triangleright S U_{a}(q)$, a even & $q^{2 a}$ & $\left(q^{a}-1\right)\left(q^{a-1}+1\right)$ & $q^{2 a-2}+q^{a}-q^{a-1}-2$ \\
& & $q^{a-1}(q-1)\left(q^{a}-1\right)$ & $q^{a-1}\left(q^{a-1}+1\right)$ \\
\hline (A6): $A_{0} \triangleright S U_{a}(q)$, a odd & $q^{2 a}$ & $\left(q^{a}+1\right)\left(q^{a-1}-1\right)$ & $q^{2 a-2}-q^{a}+q^{a-1}-2$ \\
& & $q^{a-1}(q-1)\left(q^{a}+1\right)$ & $q^{a-1}\left(q^{a-1}-1\right)$ \\
\hline (A7): $A_{0} \triangleright \Omega_{2 a}^{+}(q)$ & $q^{2 a}$ & $\left(q^{a}-1\right)\left(q^{a-1}+1\right)$ & $q^{2 a-2}+q^{a}-q^{a-1}-2$ \\
& & $q^{a-1}(q-1)\left(q^{a}-1\right)$ & $q^{a-1}\left(q^{a-1}+1\right)$ \\
\hline (A7): $A_{0} \triangleright \Omega_{2 a}^{-}(q)$ & $q^{2 a}$ & $\left(q^{a}+1\right)\left(q^{a-1}-1\right)$ & $q^{2 a-2}-q^{a}+q^{a-1}-2$ \\
& & $q^{a-1}(q-1)\left(q^{a}+1\right)$ & $q^{a-1}\left(q^{a-1}-1\right)$ \\
\hline (A8): $A_{0} \triangleright S L_{5}(q)$ & $q^{10}$ & $\left(q^{5}-1\right)\left(q^{2}+1\right)$ & $q^{5}+q^{4}-q^{2}-2$ \\
& & $q^{2}\left(q^{3}-1\right)\left(q^{5}-1\right)$ & $q^{2}\left(q^{2}+1\right)$ \\
\hline (A9): $A_{0} \triangleright B_{3}(q)$ & $q^{8}$ & $\left(q^{4}-1\right)\left(q^{3}+1\right)$ & $q^{6}+q^{4}-q^{3}-2$ \\
& & $q^{3}\left(q^{4}-1\right)(q-1)$ & $q^{3}\left(q^{3}+1\right)$ \\
\hline (A10): $A_{0} \triangleright D_{5}(q)$ & $q^{16}$ & $\left(q^{8}-1\right)\left(q^{3}+1\right)$ & $q^{8}+q^{6}-q^{3}-2$ \\
& & $q^{3}\left(q^{8}-1\right)\left(q^{5}-1\right)$ & $q^{3}\left(q^{3}+1\right)$ \\
\hline (A11): $A_{0} \triangleright S z(q)$ & $q^{4}$ & $\left(q^{2}+1\right)(q-1)$ & $q-2$ \\
& & $q\left(q^{2}+1\right)(q-1)$ & $q(q-1)$ \\
\hline
\end{tabular}


TABLE 4. Aut $(G)$ in classes $B$ of theorem

\begin{tabular}{|c|c|c|c|}
\hline$n=p^{d}$ & $R$ & $k ; l$ & $(n, k, \lambda, \mu)$ \\
\hline $2^{6}$ & $3^{1+2}$ & $27 ; 36$ & $(64,27,10,12)$ \\
\hline $3^{4}$ & $D_{8}$ or $Q_{8}$ & $32 ; 48$ & $(81,32,13,12)$ \\
\hline $7^{2}$ & $D_{8}$ or $Q_{8}$ & $24 ; 24$ & $(49,24,11,12)$ \\
\hline $13^{2}$ & $D_{8}$ or $Q_{8}$ & $72 ; 96$ & $(169,72,31,30)$ \\
\hline $17^{2}$ & $D_{8}$ or $Q_{8}$ & $96 ; 192$ & $(289,96,35,30)$ \\
\hline $19^{2}$ & $D_{8}$ or $Q_{8}$ & $144 ; 216$ & $(361,144,59,56)$ \\
\hline $23^{2}$ & $D_{8}$ or $Q_{8}$ & $264 ; 264$ & $(529,264,131,132)$ \\
\hline $3^{6}$ & $D_{8}$ or $Q_{8}$ & $104 ; 624$ & $(729,104,31,12)$ \\
\hline $29^{2}$ & $D_{8}$ or $Q_{8}$ & $168 ; 672$ & $(841,168,47,30)$ \\
\hline $31^{2}$ & $D_{8}$ or $Q_{8}$ & $240 ; 720$ & $(961,240,71,56)$ \\
\hline $47^{2}$ & $D_{8}$ or $Q_{8}$ & $1104 ; 1104$ & $(2209,1104,551,552)$ \\
\hline $3^{4}$ & $R_{1}^{2}$ & $32 ; 48$ & $(81,32,13,12)$ \\
\hline $3^{4}$ & $R_{2}^{2}$ & $16 a ; 16 b(a+b=5)$ & $(81,16,7,2)$ or $(81,32,13,12)$ \\
\hline $5^{4}$ & $R_{2}^{2}$ & $240 ; 384$ & $(625,240,95,90)$ \\
\hline $5^{4}$ & $R_{3}^{2}$ & $240 ; 384$ & $(625,240,95,90)$ \\
\hline $7^{4}$ & $R_{2}^{2}$ & $480 ; 1920$ & $(2401,480,119,90)$ \\
\hline $3^{8}$ & $R_{2}^{3}$ & $1440 ; 5120$ & $(6561,1440,351,306)$ \\
\hline \hline
\end{tabular}

TABLE 5. Aut $(G)$ in class $C$ of theorem

\begin{tabular}{|c|c|c|c|}
\hline$n=p^{d}$ & $L$ & $k ; l$ & $(n, k, \lambda, \mu)$ \\
\hline $3^{4}$ & $A_{5}$ & $40 ; 40$ & $(81,40,19,20)$ \\
\hline $31^{2}$ & $A_{5}$ & 360,600 & $(961,360,119,110)$ \\
\hline $41^{2}$ & $A_{5}$ & 480,1200 & $(1681,480,149,132)$ \\
\hline $7^{4}$ & $A_{5}$ & $960 ; 1440$ & $(2401,960,389,380)$ \\
\hline $71^{2}$ & $A_{5}$ & 840,4200 & $(5041,840,179,132)$ \\
\hline $79^{2}$ & $A_{5}$ & 1560,4680 & $(6241,1560,419,380)$ \\
\hline $89^{2}$ & $A_{5}$ & 2640,5280 & $(7921,2640,899,870)$ \\
\hline $2^{6}$ & $A_{6}$ & $18 ; 45$ & $(64,18,2,6)$ \\
\hline $5^{4}$ & $A_{6}$ & $144 ; 480$ & $(625,144,43,30)$ \\
\hline $2^{8}$ & $A_{7}$ & $45 ; 210$ & $(256,45,16,6)$ \\
\hline $7^{4}$ & $A_{7}$ & $720 ; 1680$ & $(2401,720,229,210)$ \\
\hline $2^{8}$ & $A_{9}$ & $105 ; 150$ & $(256,105,44,42)$ \\
\hline $2^{8}$ & $A_{10}$ & $45 ; 210$ & $(256,45,16,6)$ \\
\hline $2^{8}$ & $L_{2}(17)$ & $102 ; 153$ & $(256,102,38,42)$ \\
\hline $3^{6}$ & $L_{3}(4)$ & $224 ; 504$ & $(729,224,61,72)$ \\
\hline $7^{4}$ & $U_{4}(2)$ & $240 ; 2160$ & $(2401,240,59,20)$ \\
\hline $3^{5}$ & $M_{11}$ & $22 ; 220$ & $(243,22,1,2)$ \\
\hline $3^{5}$ & $M_{11}$ & $110 ; 132$ & $(243,110,37,60)$ \\
\hline $2^{11}$ & $M_{24}$ & $276 ; 1771$ & $(2048,276,44,36)$ \\
\hline $2^{11}$ & $M_{24}$ & $759 ; 1288$ & $(2048,759,310,264)$ \\
\hline $3^{12}$ & Suz and $G_{2}(4)$ & $65520 ; 465920$ & $\left(3^{12}, 65520,8559,8010\right)$ \\
\hline $2^{12}$ & $J_{2}$ & $1575 ; 2520$ & $(4096,1575,614,600)$ \\
\hline $5^{6}$ & $J_{2}$ & 7560,8064 & $(15625,7560,3655,3660)$ \\
\hline & & & \\
\hline
\end{tabular}

\section{PRIMitive DTGS OF DiAMETER TWO OF SIMPle SOCLE TYPE}

Using the complete classification of the simple socle type rank three groups, we will be able to list all their corresponding primitive distance-transitive graphs of diameter two. 
The classification of finite simple groups can be invoked to make further subdivision of the possibilities for $F^{*}(A)$.
$\begin{cases}(i) & \text { Alternating groups; } \\ (\text { ii }) & \text { Groups of Lie type; } \\ \text { (iii) } & \text { Sporadic groups. }\end{cases}$

5.1. The alternating simple socle. The complete list of primitive representations of rank three of alternating groups given by Eiichi Bannai (see [8, table 2]) together with the result of Martin W. Liebeck, Cheryl E. Praeger and Jan Saxl (see [23]) enable us to have the following theorem.

Theorem 5.3. Let $A$ be a finite primitive rank three permutation group of degree $n$. Assume that the socle $L$ of $A$ is an alternating group, and let $H$ be the stabilizer in $L$ of a point. Then $L$ is as in Table 6 below; so are the subdegrees $k, l$ and the corresponding strongly regular graph.

TABLE 6. Primitive distance-transitive actions of rank three alternating group

\begin{tabular}{|c|c|c|c|}
\hline$L$ & $n$ & $k ; l$ & graph \\
\hline$A_{3}$ & 3 & $1 ; 1$ & $K_{3}$ \\
\hline$A_{n}, n \geq 5$ & $\frac{n(n-1)}{2}$ & $2(n-2) ;$ & $J(n, 2)$ \\
\hline$A_{4}$ & 3 & $1 ; 1$ & $K_{3}$ \\
\hline$A_{6}$ & 15 & $8 ; 6$ & $J(6,2)$ \\
\hline$A_{8}$ & 35 & $16 ; 18$ & $\mathrm{~A} J(8,4)^{*}$ \\
\hline$A_{9}$ & 120 & $56 ; 63$ & $\Sigma_{120}$ \\
\hline$A_{10}$ & 126 & 25,100 & $\mathrm{~A} J(10,5)^{* *}$ \\
\hline
\end{tabular}

* The quotient Johnson graph A $J(8,4)$.

** The quotient Johnson graph A $J(10,5)$.

\subsection{The simple socle of Lie type.}

5.2.1. The classical groups. In [19], W. M. Kantor and R. A. Liebler gave the complete list of the finite primitive permutation classical groups of rank three together with theirs subdegrees of the two orbits. Hence we do have tables $7 \& 8$ below.

TABLE 7. Primitive distance-transitive action of rank three classical group: exceptional classes

\begin{tabular}{|c|c|c|c|c|}
\hline $\mathrm{L}$ & $\mathrm{H}$ & $\mathrm{n}$ & $\mathrm{k} ; \mathrm{l}$ & $G=(n, k, \lambda, \mu)$ \\
\hline \hline$L_{2}(8): 3$ & $7: 6$ & 36 & $14 ; 21$ & $(36,14,7,4)$ \\
\hline$L_{3}(4)$ & $A_{6}$ & 56 & $10 ; 45$ & $(56,10,0,2)$ \\
\hline$S_{6}(2)$ & $G_{2}(2)$ & 120 & $56 ; 63$ & $(120,56,28,24)$ \\
\hline$O_{7}(3)$ & $G_{2}(3)$ & 1080 & $351 ; 728$ & $(1080,351,126,108)$ \\
\hline$U_{3}(3): 2$ & $L_{3}(2): 2$ & 36 & $14 ; 21$ & $(36,14,4,6)$ \\
\hline$U_{3}(5)$ & $A_{7}$ & 50 & $7 ; 42$ & $(50,7,0,1)$ \\
\hline$U_{4}(3)$ & $L_{3}(4)$ & 162 & $56 ; 105$ & $(162,56,10,24)$ \\
\hline$U_{6}(2)$ & $U_{4}(3): 2$ & 1408 & $567 ; 840$ & $(1408,567,246,216)$ \\
\hline
\end{tabular}

(*) Notice that the parameters $\mu$ of the polar graph $N O_{2 n}^{ \pm}(3)$ in $O_{2 n}^{ \pm}(3)$ and $l$ of the polar graph $N O_{2 n+1}^{ \pm}(8)$ in $O_{2 n+1}(8): 3$ (Table 8 below) are not correct in Tables 21 and 22, resp. of [11]. 
TABLE 8. Primitive rank three classical group: Infinite classes

\begin{tabular}{|c|c|c|c|c|}
\hline Group & $n$ & Remark & $\begin{array}{l}k \\
l\end{array}$ & $\begin{array}{l}\lambda \\
\mu\end{array}$ \\
\hline 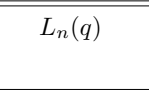 & $\begin{array}{l}\frac{\left(q^{n+1}-1\right)\left(q^{n}-1\right)}{(q+1)(q-1)^{2}} \\
\end{array}$ & $n \geq 4$ & $\begin{array}{c}\frac{q(q+1)\left(q^{n-1}-1\right)}{q-1} \\
\frac{q^{4}\left(q^{n-1}-1\right)\left(q^{n-2}-1\right)}{(q+1)(q-1)^{2}}\end{array}$ & $\begin{array}{c}\frac{q^{n}-1}{q-1}+q^{2}-2 \\
(q+1)^{2}\end{array}$ \\
\hline$S_{2 n}(q)$ & $\frac{q^{2 n}-1}{q-1}$ & $n \geq 2$ & $\begin{array}{c}q \frac{q^{2 n-2}-1}{q-1} \\
q^{2 n-1}\end{array}$ & $\begin{array}{l}\frac{q^{2 n-2}-1}{q-1}-2 \\
\frac{q^{2 n-2}-1}{q-1}\end{array}$ \\
\hline$O_{2 n+1}(q)$ & $\frac{q^{2 n}-1}{q-1}$ & $n \geq 2$ & $\begin{array}{c}q \frac{q^{2 n-2}-1}{q-1} \\
q^{2 n-1}\end{array}$ & $\begin{array}{l}\frac{q^{2 n-2}-1}{q-1}-2 \\
\frac{q^{2 n-2}-1}{q-1}\end{array}$ \\
\hline$O_{2 n}^{ \pm}(q)$ & $\frac{\left(q^{n} \mp 1\right)\left(q^{n-1} \pm 1\right)}{q-1}$ & $n \geq 3$ & $\begin{array}{c}\frac{q\left(q^{n-1} \mp 1\right)\left(q^{n-2} \pm 1\right)}{q-1} \\
q^{2 n-2}\end{array}$ & $\begin{array}{c}\frac{q\left(q^{n-2} \mp 1\right)\left(q^{n-2} \pm q\right)}{q-1}+q-1 \\
\frac{\left(q^{n-1} \mp 1\right)\left(q^{n-2} \pm 1\right)}{q-1}\end{array}$ \\
\hline$O_{10}^{+}(q)$ & $\frac{\left(q^{8}-1\right)\left(q^{3}+1\right)}{q-1}$ & & $\begin{array}{c}\frac{q\left(q^{5}-1\right)\left(q^{2}+1\right)}{q-1} \\
\frac{q^{6}\left(q^{5}-1\right)}{q-1}\end{array}$ & $\begin{array}{l}\frac{q^{2}\left(q^{3}-1\right)(q+1)}{q-1}+q-1 \\
\quad \frac{\left(q^{3}-1\right)\left(q^{2}+1\right)}{q-1}\end{array}$ \\
\hline$O_{2 n}^{ \pm}(2)$ & $2^{2 n-1} \pm 2^{n-1}$ & $n \geq 3$ & $\begin{array}{c}2^{2 n-2}-1 \\
2^{2 n-2} \pm 2^{n-1}\end{array}$ & $\begin{array}{c}2^{2 n-3}-2 \\
2^{2 n-3} \mp 2^{n-2}\end{array}$ \\
\hline$O_{2 n+1}(3)$ & $\frac{3^{n}\left(3^{n} \pm 1\right)}{2}$ & $n \geq 2$ & $\begin{array}{c}\frac{3^{n-1}\left(3^{n} \mp 1\right)}{2} \\
3^{2 n-1} \pm 2.3^{n-1}-1\end{array}$ & $\begin{array}{l}\frac{3^{n-1}\left(3^{n-1} \mp 1\right)}{2} \\
\frac{3^{n-1}\left(3^{n-1} \mp 1\right)}{2}\end{array}$ \\
\hline$O_{2 n}^{ \pm}(3)$ & $\frac{3^{n-1}\left(3^{n} \mp 1\right)}{2}$ & $n \geq 3$ & $\begin{array}{c}3^{n-1} \cdot \frac{3^{n-1} \mp 1}{2} \\
3^{2 n-2}-1\end{array}$ & $\begin{array}{l}3^{n-2} \cdot \frac{3^{n-1} \pm 1}{2} \\
3^{n-1} \cdot \frac{3^{n-2} \mp 1}{2} *\end{array}$ \\
\hline$O_{2 n+1}(4)$ & $2^{2 n-1}\left(2^{2 n} \mp 1\right)$ & $n \geq 2$ & $\begin{array}{c}\left(2^{2 n} \pm 1\right)\left(2^{2 n-2} \mp 1\right) \\
2^{2 n-2}\left(2^{2 n} \pm 1\right)\end{array}$ & $\begin{array}{c}2^{4 n-3} \mp 3.2^{2 n-2}-2 \\
2^{2 n-1}\left(2^{2 n-2} \mp 1\right)\end{array}$ \\
\hline$O_{2 n+1}(8): 3$ & $2^{3 n-1}\left(2^{3 n} \mp 1\right)$ & $n \geq 2$ & $\begin{array}{l}\left(2^{3 n-3} \pm 1\right)\left(2^{3 n} \mp 1\right) \\
\left(3.2^{6 n-3} \mp 11.2^{3 n-3}\right)^{*}\end{array}$ & $\begin{array}{c}2^{6 n-5} \mp 2^{3 n-3} \pm 2^{3 n}-2 \\
2^{3 n-2}\left(2^{3 n-3} \pm 1\right)\end{array}$ \\
\hline$U_{2 n+1}(q)$ & $\frac{\left(q^{2 n}-1\right)\left(q^{2 n+1}+1\right)}{q^{2}-1}$ & $n \geq 1$ & $\begin{array}{c}\frac{q^{2}\left(q^{2 n-2}-1\right)\left(q^{2 n-1}+1\right)}{q^{2}-1} \\
q^{4 n-1}\end{array}$ & $\begin{array}{c}\frac{q^{3}\left(q^{2 n-3}+1\right)\left(q^{2 n-3}-q\right)}{q^{2}-1}+q^{2}-1 \\
\frac{\left(q^{2 n-2}-1\right)\left(q^{2 n-1}+1\right)}{q^{2}-1}\end{array}$ \\
\hline$U_{2 n}(q)$ & $\frac{\left(q^{2 n}-1\right)\left(q^{2 n-1}+1\right)}{q^{2}-1}$ & $n \geq 2$ & $\begin{array}{c}\frac{q^{2}\left(q^{2 n-2}+1\right)\left(q^{2 n-1}-1\right)}{q^{2}-1} \\
q^{4 n-1}\end{array}$ & $\begin{array}{c}\frac{q^{3}\left(q^{2 n-3}-1\right)\left(q^{2 n-3}+q\right)}{q^{2}-1}+q^{2}-1 \\
\frac{\left(q^{2 n-2}+1\right)\left(q^{2 n-1}-1\right)}{q^{2}-1}\end{array}$ \\
\hline$U_{5}(q)$ & $\left(q^{5}+1\right)\left(q^{3}+1\right)$ & & $\begin{array}{c}q^{3}\left(q^{2}+1\right) \\
q^{8}\end{array}$ & $\begin{array}{l}q^{3}-1 \\
q^{2}+1 \\
\end{array}$ \\
\hline$U_{2 n+1}(2)$ & $\frac{2^{2 n}\left(2^{2 n+1}+1\right)}{3}$ & $n \geq 2$ & $\begin{array}{c}\left(2^{2 n}-1\right)\left(2^{2 n-1}+1\right) \\
2^{2 n-1} \cdot \frac{2^{2 n}-1}{3}\end{array}$ & $\begin{array}{c}3.2^{4 n-3}+2^{2 n-1}-2 \\
3.2^{2 n-2}\left(2^{2 n-1}+1\right)\end{array}$ \\
\hline$U_{2 n}(2)$ & $\frac{2^{2 n-1}\left(2^{2 n}-1\right)}{3}$ & $n \geq 2$ & $\begin{array}{c}\left(2^{2 n-1}+1\right)\left(2^{2 n-2}-1\right) \\
2^{2 n-2} \cdot \frac{2^{2 n-1}+1}{3}\end{array}$ & $\begin{array}{l}3.2^{4 n-5}-2^{2 n-2}-2 \\
3.2^{2 n-3}\left(2^{2 n-2}-1\right)\end{array}$ \\
\hline$E_{6}(q)$ & $\frac{\left(q^{12}-1\right)\left(q^{9}-1\right)}{\left(q^{4}-1\right)(q-1)}$ & & $\begin{array}{l}\frac{q\left(q^{8}-1\right)\left(q^{3}+1\right)}{(q-1)} \\
\frac{q^{8}\left(q^{5}-1\right)\left(q^{4}+1\right)}{(q-1)} \\
\end{array}$ & $\begin{array}{c}\frac{q^{9}+q^{7}-q^{4}-2 q+1}{q-1} \\
\frac{\left(q^{4}-1\right)\left(q^{3}+1\right)}{q-1} \\
\end{array}$ \\
\hline
\end{tabular}

5.2.2. The exceptional groups. In [22], Martin W. Liebeck and Jan Saxl proved the following result.

Theorem 5.4. Let $A$ be a finite primitive rank three permutation group of degree $n$. Assume that the socle $L$ of $A$ is an exceptional simple group of Lie type, and let $H$ be the stabilizer in $L$ of a point. Then $L$ and the subdegrees $k, l$ are as in table 9 below.

5.3. The sporadic simple socle. In [22], Liebeck and Saxl mention that A. Brouwer gave them the complete list of the finite primitive permutation sporadic groups of rank three together with theirs subdegrees of the two orbits. Hence we do have the following result.

Theorem 5.5. Let $A$ be a group satisfying $T \leq A \leq A u t(T)$ where $T$ is a sporadic simple group. Suppose that $A$ acts distance-transitively on a primitive graph $G$ of diameter two and the permutation action of $A$ on the vertex set of $G$ is similar to its action on the cosets of a subgroup $B$. Then either $T$ also acts distance-transitively on $G$ and the possibilities for $T$ and $T \cap B$ are given in the table 10 below, or $A \cong A u t\left(J_{2}\right), B \cong 2_{-}^{1+4}: S_{5}$ (also in the table). The graph $G$ is determined up to complement. 
TABLE 9. Primitive distance-transitive actions of rank three exceptional group of Lie type

\begin{tabular}{|c|c|c|c|}
\hline$L$ & $n$ & $k ; l$ & $(n, k, \lambda, \mu)$ \\
\hline$G_{2}(3)$ & 351 & $126 ; 224$ & $(351,126,45,45)$ \\
\hline$G_{2}(4)$ & $\begin{array}{c}416 \\
2016\end{array}$ & $\begin{array}{c}100 ; 315 \\
975 ; 1040\end{array}$ & $\begin{array}{c}(416,100,36,20) \\
(2016,975,462,480)\end{array}$ \\
\hline$G_{2}(8)$ & 130816 & $32319 ; 98496$ & $(130816,32319,7742,8064)$ \\
\hline$E_{6}(q)$ & $\frac{\left(q^{12}-1\right)\left(q^{9}-1\right)}{\left(q^{4}-1\right)(q-1)}$ & $\begin{array}{l}\frac{q\left(q^{8}-1\right)\left(q^{3}+1\right)}{(q-1)} ; \\
\frac{q^{8}\left(q^{5}-1\right)\left(q^{4}+1\right)}{(q-1)}\end{array}$ & $\left(n, k, \frac{q^{9}+q^{7}-q^{4}-2 q+1}{q-1}, \frac{\left(q^{4}-1\right)\left(q^{3}+1\right)}{q-1}\right)$ \\
\hline
\end{tabular}

TABLE 10. Primitive distance-transitive actions of rank three sporadic groups

\begin{tabular}{|c|c|c|c|c|}
\hline Group & $n$ & $k ; l$ & Graph & $(n, k, \lambda, \mu)$ \\
\hline$M_{11}$ & 55 & $18 ; 36$ & $J(11,2)$ & $(55,18,9,4)$ \\
\hline$M_{12}$ & 66 & $20 ; 45$ & $J(12,2)$ & $(77,60,47,45)$ \\
\hline$M_{22}$ & 77 & $60 ; 16$ & & $(176,70,18,34)$ \\
& 176 & $70 ; 105$ & & $(253,42,21,4)$ \\
\hline$M_{23}$ & 253 & $42 ; 210$ & $J(23,2)$ & $(253,112,36,60)$ \\
& 253 & $112 ; 140$ & & $(276,44,22,4)$ \\
\hline$M_{24}$ & 276 & $44 ; 231$ & $J(24,2)$ & $(1288,792,476,504)$ \\
& 1288 & $792 ; 495$ & & $(100,36,14,12)$ \\
\hline$J_{2}$ & 100 & $36 ; 63$ & Hall-Janko graph & $(100,77,60,56)$ \\
\hline$H S$ & 100 & $77 ; 22$ & Higman-Sims graph & $(1782,416,100,96)$ \\
\hline$M c L$ & 275 & $162 ; 112$ & McLaughlin graph & $(2300,1408,840,896)$ \\
\hline$S u z$ & 1782 & $416 ; 1365$ & Suzuki graph & $(4060,1755,730,780)$ \\
\hline$C o_{2}$ & 2300 & $1408 ; 891$ & & $(3510,693,180,126)$ \\
\hline$R u$ & 4060 & $1755 ; 2304$ & Rudvalis graph & $(14080,3159,918,648)$ \\
\hline$F i_{22}$ & 3510 & $693 ; 2816$ & 3-transpositions & $(31671,3510,693,351)$ \\
& 14080 & $3159 ; 10920$ & & $(137632,28431,6030,5832)$ \\
\hline$F i_{23}$ & 31671 & $3510 ; 28160$ & 3-transpositions & $(306936,31671,3510,3240)$ \\
\hline$F i_{24}^{\prime}$ & 137632 & $28431 ; 109200$ & & 3-transpositions \\
\hline
\end{tabular}

\section{PRIMITIVE DISTANCE-TRANSITIVE GRAPHS OF DIAMETER TWO}

In Table 11 below we give a complete list of all primitive distance-transitive graphs $G$ of diameter two (except those arise from one dimensional affine groups, see [26, 31]) with their complements. The appropriate section for the description of each graph being listed is in the final column of the table.

Notice that graphs for $S U_{a}(q)$ (affine polar graphs $\left.V O_{2 a}^{ \pm}(q)\right) \subseteq$ graphs for $\Omega_{2 a}^{+}(q) \cup$ graphs for $\Omega_{2 a}^{-}(q)$ 


\section{TABLE 11. Primitive distance-transitive graphs of diameter two}

\begin{tabular}{|c|c|}
\hline $\begin{aligned} G & =(n, k, \lambda, \mu) \\
\bar{G} & =(n, \bar{k}, \bar{\lambda}, \bar{\mu})\end{aligned}$ & Section \\
\hline $\begin{array}{c}\text { Hamming graphs } H(2, q), q>2 \\
\bar{H}(2, q), q>2\end{array}$ & 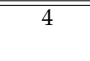 \\
\hline $\begin{array}{c}\text { Generalized quadrangle } G Q\left(p^{m}-1,1\right)=\left(p^{2 m}, 2\left(p^{m}-1\right), p^{m}-2,2\right) \\
\overline{G Q}\left(p^{m}-1,1\right)\end{array}$ & 4 \\
\hline Bilinear forms $H_{q}(m, 2)\left(q^{2 m}, \frac{\left.(q+1)\left(q^{m}-1\right), q^{m}+q^{2}-q-2, q(q+1)\right)}{H_{q}(m, 2)}\right.$ & 4 \\
\hline $\begin{aligned} \text { Orthogonal array } O A\left(q+1, q^{m}\right)= & \left(q^{2 m},(q+1)\left(q^{m}-1\right), q^{m}+q^{2}-q-2, q(q+1)\right) \\
& O A\left(q+1, q^{m}\right)\end{aligned}$ & 4 \\
\hline $\begin{array}{c}\text { Affine polar } V O_{2 a}^{+}(q)=\left(q^{2 a},\left(q^{a}-1\right)\left(q^{a-1}+1\right), q^{2 a-2}+q^{a}-q^{a-1}-2, q^{a-1}\left(q^{a-1}+1\right)\right) \\
\overline{V O}_{2 a}^{+}(q)\end{array}$ & 4 \\
\hline $\begin{array}{c}\text { Af fine polar } V O_{2 a}^{-}(q)=\left(q^{2 a},\left(q^{a}+1\right)\left(q^{a-1}-1\right), q^{2 a-2}-q^{a}+q^{a-1}-2, q^{a-1}\left(q^{a-1}-1\right)\right) \\
\overline{V O_{2 a}^{-}(q)}\end{array}$ & 4 \\
\hline $\begin{array}{c}\text { Orthogonal array } O A\left(q^{a-1}+1, q^{a}\right)=\left(q^{2 a},\left(q^{a}-1\right)\left(q^{a-1}+1\right), q^{2 a-2}+q^{a}-q^{a-1}-2, q^{a-1}\left(q^{a-1}+1\right)\right) \\
\overline{O A}\left(q^{a-1}+1, q^{a}\right)\end{array}$ & 4 \\
\hline Negative Latin Square $\left(q^{2 a}, \frac{\left(q^{a}+1\right)\left(q^{a-1}-1\right), q^{2 a-2}-q^{a}}{\text { negative }- \text { Latin }- \text { square }}+q^{a-1}-2, q^{a-1}\left(q^{a-1}-1\right)\right)$ & 4 \\
\hline Alternating forms Alt $(5, q)=\left(q^{10}, \frac{\left.\left(q^{5}-1\right)\left(q^{2}+1\right), q^{5}+q^{4}-q^{2}-2, q^{2}\left(q^{2}+1\right)\right)}{\operatorname{Alt}(5, q)}\right.$ & 4 \\
\hline $\begin{aligned} \text { Orthogonal array } O A\left(q^{3}+1, q^{4}\right)= & =\left(q^{8},\left(q^{4}-1\right)\left(q^{3}+1\right), q^{6}+q^{4}-q^{3}-2, q^{3}\left(q^{3}+1\right)\right) \\
O A & \left(q^{3}+1, q^{4}\right)\end{aligned}$ & 4 \\
\hline $\begin{aligned} \text { Orthogonal array } O A\left(q^{3}+1, q^{8}\right)= & \frac{\left(q^{16},\left(q^{8}-1\right)\left(q^{3}+1\right), q^{8}+q^{6}-q^{3}-2, q^{3}\left(q^{3}+1\right)\right)}{O A}\left(q^{3}+1, q^{8}\right)\end{aligned}$ & 4 \\
\hline $\begin{array}{c}\text { Hermitean forms } \operatorname{Her}\left(2, q^{2}\right)=\left(q^{4},\left(q^{2}+1\right)(q-1), q-2, q(q-1)\right) \\
\operatorname{Her}\left(2, q^{2}\right)\end{array}$ & 4 \\
\hline $\begin{aligned} \text { Triangular graphs } T(n) & =\left(\frac{n(n-1)}{2}, 2(n-2),(n-2), 4\right) \\
\bar{T}(n) & \end{aligned}$ & 5.1 \\
\hline$E_{6,1}=\left(\frac{\left(q^{12}-1\right)\left(q^{9}-1\right)}{\left(q^{4}-1\right)(q-1)}, \frac{q\left(q^{8}-1\right)\left(q^{3}+1\right)}{(q-1)}, \frac{q^{9}+q^{7}-q^{4}-2 q+1}{q-1}, \frac{\left(q^{4}-1\right)\left(q^{3}+1\right)}{q-1}\right)$ & 5.2 \\
\hline Grassmann $J_{q}(n+1,2)$ & 5.2 \\
\hline$B_{n, 1}(q) ;$ Polar graphs in $O_{2 n+1}(q)$ & 5.2 \\
\hline $\begin{array}{c}C_{n, 1}(q) ; \text { Polar graphs in } \operatorname{Sp}_{2 n}(q) \\
\overline{C_{n, 1}(q)}\end{array}$ & 5.2 \\
\hline$D_{n, 1}(q) ;$ Polar graphs in $O_{2 n}^{+}(q)$ & 5.2 \\
\hline${ }^{2} D_{n, 1}(q) ;$ Polar graphs in $O_{2 n}^{-}(q)$ & 5.2 \\
\hline $\begin{array}{l}\text { half dual Polar graph } D_{5,5}(q) \\
\qquad D_{5,5}(q)\end{array}$ & 5.2 \\
\hline $\mathrm{NO}_{2 n}^{-}(2) ;$ Polar graphs in $\mathrm{O}_{2 n}^{+}(2)$ & 5.2 \\
\hline$N O_{2 n}^{+}(2) ;$ Polar graphs in $O_{2 n}^{-}(2)$ & 5.2 \\
\hline$N O_{2 n+1}^{ \pm}(3) ; \frac{\text { Polar graphs in } O_{2 n+1}(3)}{N O_{2 n+1}^{ \pm}(3)}$ & 5.2 \\
\hline$N O_{2 n}^{ \pm}(3) ;$ Polar graphs in $O_{2 n}^{ \pm}(3)$ & 5.2 \\
\hline$N O_{2 n+1}^{\mp}(4) ; \frac{\text { Polar graphs in } O_{2 n+1}^{ \pm}(4)}{N O_{2 n+1}^{\mp}(4)}$ & 5.2 \\
\hline
\end{tabular}

\begin{tabular}{|c|c|c|}
\hline$G=(n, k, \lambda, \mu)$ & $\bar{G}=(n, \bar{k}, \bar{\lambda}, \bar{\mu})$ & Section \\
\hline$N O_{2 n+1}^{ \pm}(8) ;$ Polar graphs in $O_{2 n+1}^{ \pm}(8)$ & $5.2 \overline{N O_{2 n+1}^{ \pm}(8)}$ & 5.2 \\
\hline${ }^{2} A_{2 n, 1}(q) ;$ Polar graphs in $U_{2 n+1}\left(q^{2}\right)$ & $5.2^{2} A_{2 n, 1}(q)$ & 5.2 \\
\hline${ }^{2} A_{2 n-1,1}(q) ;$ Polar graphs in $U_{2 n}\left(q^{2}\right)$ & $\overline{2} A_{2 n-1,1}(q)$ & 5.2 \\
\hline Dual Polar graph $\left[{ }^{2} A_{4}(q)\right]$ & $\overline{\left[{ }^{2} A_{4}(q)\right]}$ & 5.2 \\
\hline$N U_{2 n+1}(2) ;$ Polar graphs in $U_{2 n+1}(2)$ & $\overline{N U_{2 n+1}(2)}$ & 5.2 \\
\hline$N U_{2 n}(2) ;$ Polar graphs in $U_{2 n}(2)$ & $\overline{N U_{2 n}(2)}$ & 5.2 \\
\hline
\end{tabular}


Distance-transitive strongly regular graphs

\begin{tabular}{|c|c|c|}
\hline$G=(n, k, \lambda, \mu)$ & $\bar{G}=(n, \bar{k}, \bar{\lambda}, \bar{\mu})$ & Section \\
\hline$(64,27,10,12)$ & $(64,36,20,20)$ & 4 \\
\hline$(81,32,13,12)$ & $(81,48,27,30)$ & 4 \\
\hline$(49,24,11,12)$ & $(49,24,11,12)$ & 4 \\
\hline$(169,72,31,30)$ & $(169,96,53,56)$ & 4 \\
\hline$(289,96,35,30)$ & $(289,192,125,132)$ & 4 \\
\hline$(361,144,59,56)$ & $(361,216,127,132)$ & 4 \\
\hline$(529,264,131,132)$ & $(529,264,131,132)$ & 4 \\
\hline$(729,104,31,12)$ & $(729,624,531,552)$ & 4 \\
\hline$(841,168,47,30)$ & $(841,672,533,552)$ & 4 \\
\hline$(961,240,71,56)$ & $(961,720,535,552)$ & 4 \\
\hline$(2209,1104,551,552)$ & $(2209,1104,551,552)$ & 4 \\
\hline$(81,16,7,2)$ & $(81,64,49,56)$ & 4 \\
\hline$(625,240,95,90)$ & $(625,384,233,240)$ & 4 \\
\hline$(2401,480,119,90)$ & $(2401,1920,1529,1560)$ & 4 \\
\hline$(6561,1440,351,306)$ & $(6561,5120,3985,4032)$ & 4 \\
\hline$(81,40,19,20)$ & $(81,40,19,20)$ & 4 \\
\hline$(961,360,119,110)$ & $(961,600,349,360)$ & 4 \\
\hline$(1681,480,149,132)$ & $(1681,1200,851,870)$ & 4 \\
\hline$(2401,960,389,380)$ & $(2401,1440,859,870)$ & 4 \\
\hline$(5041,840,179,132)$ & $(5041,4200,3492,3540)$ & 4 \\
\hline$(6241,1560,419,380)$ & $(6241,4680,3499,3540)$ & 4 \\
\hline$(7921,2640,899,870)$ & $(7921,5280,3509,3540)$ & 4 \\
\hline$(64,18,2,6)$ & $(64,45,32,30)$ & 4 \\
\hline$(625,144,43,30)$ & $(625,480,365,380)$ & 4 \\
\hline$(256,45,16,6)$ & $(256,210,170,182)$ & 4 \\
\hline$(2401,720,229,210)$ & $(2401,1680,1169,1190)$ & 4 \\
\hline$(256,105,44,42)$ & $(256,150,86,90)$ & 4 \\
\hline$(256,102,38,42)$ & $(256,153,92,90)$ & 4 \\
\hline$(729,224,61,72)$ & $(729,504,351,342)$ & 4 \\
\hline$(2401,240,59,20)$ & $(2401,2160,1939,1980)$ & 4 \\
\hline$(243,22,1,2)$ & $(243,220,199,200)$ & 4 \\
\hline$(243,110,37,60)$ & $(243,132,81,60)$ & 4 \\
\hline$(2048,276,44,36)$ & $(2048,1771,1530,1540)$ & 4 \\
\hline$(2048,759,310,264)$ & $(2048,1288,792,840)$ & 4 \\
\hline$\left(3^{12}, 65520,8559,8010\right)$ & $\left(33^{12}, 465920,408409,408960\right)$ & 4 \\
\hline$(4096,1575,614,600)$ & $(4096,2520,1544,1560)$ & 4 \\
\hline$(15625,7560,3655,3660)$ & $(15625,8064,4163,4160)$ & 4 \\
\hline & & \\
\hline & & 4 \\
\hline
\end{tabular}


M. R. Alfuraidan

\begin{tabular}{|c|c|c|}
\hline$G=(n, k, \lambda, \mu)$ & $\bar{G}=(n, \bar{k}, \bar{\lambda}, \bar{\mu})$ & Section \\
\hline $\mathrm{A} J(8,4)=(35,16,6,8)$ & $(35,18,9,9)$ & 5.1 \\
\hline$\Sigma_{120}=(120,56,28,24)$ & $(120,63,30,36)$ & 5.1 \\
\hline $\mathrm{A} J(10,5)=(126,25,8,4)$ & $(126,100,78,84)$ & 5.1 \\
\hline$(36,14,7,4)$ & $(36,21,10,15)$ & 5.2 \\
\hline$(56,10,0,2)$ & $(56,45,36,36)$ & 5.2 \\
\hline$(1080,351,126,108)$ & $(1080,728,484,504)$ & 5.2 \\
\hline$(36,14,4,6)$ & $(36,21,12,12)$ & 5.2 \\
\hline$(50,7,0,1)$ & $(50,42,35,36)$ & 5.2 \\
\hline$(162,56,10,24)$ & $(162,105,72,60)$ & 5.2 \\
\hline$(1408,567,246,216)$ & $(1408,840,488,520)$ & 5.2 \\
\hline$(351,126,45,45)$ & $(351,224,142,144)$ & 5.2 \\
\hline$(416,100,36,20)$ & $(416,315,234,252)$ & 5.2 \\
\hline$(2016,975,462,480)$ & $(2016,1040,544,528)$ & 5.2 \\
\hline$(130816,32319,7742,8064)$ & $(130816,98496,74240,73920)$ & 5.2 \\
\hline$(77,60,47,45)$ & $(77,16,0,4)$ & 5.3 \\
\hline$(176,70,18,34)$ & $(176,105,68,54)$ & 5.3 \\
\hline$(253,112,36,60)$ & $(253,140,87,65)$ & 5.3 \\
\hline$(1288,792,476,504)$ & $(1288,495,206,180)$ & 5.3 \\
\hline $\begin{array}{c}\text { Hall-Janko } \\
(100,36,14,12)\end{array}$ & $(100,63,38,42)$ & 5.3 \\
\hline $\begin{array}{l}\text { Higman-Sims } \\
(100,77,60,56)\end{array}$ & $(100,22,0,6)$ & 5.3 \\
\hline $\begin{array}{c}\text { McLaughlin } \\
(275,162,105,81)\end{array}$ & $(275,112,30,56)$ & 5.3 \\
\hline $\begin{array}{c}\text { Suzuki } \\
(1782,416,100,96)\end{array}$ & $(1782,1365,1044,1050)$ & 5.3 \\
\hline$(2300,1408,840,896)$ & $(2300,891,378,324)$ & 5.3 \\
\hline $\begin{array}{c}\text { Rudvalis } \\
(4060,1755,730,780)\end{array}$ & $(4060,2304,1328,1280)$ & 5.3 \\
\hline $\begin{array}{c}\text { 3-transpositions } \\
(3510,693,180,126)\end{array}$ & $(3510,2816,2248,2304)$ & 5.3 \\
\hline$(14080,3159,918,648)$ & $(14080,10920,8408,8680)$ & 5.3 \\
\hline $\begin{array}{c}\text { 3-transpositions } \\
(31671,3510,693,351)\end{array}$ & $(31671,28160,25000,25344)$ & 5.3 \\
\hline$(137632,28431,6030,5832)$ & $(137632,109200,86600,86800)$ & 5.3 \\
\hline $\begin{array}{c}\text { 3-transpositions } \\
(306936,31671,3510,3240)\end{array}$ & $(306936,275264,246832,247104)$ & 5.3 \\
\hline
\end{tabular}

\section{OPEN PROBLEM}

One of the most tantalizing classes of strongly regular graphs is the class of triangle free strongly regular graphs, or tfSRG for short. This condition can be expressed in terms of parameters as $\lambda=0$. Apart from the degenerate cases of the complete graph $K_{n}$ and the complete bipartite graph $K_{n, n}$, only seven tfSRGs are known, and these include some of the most famous graphs. The list of the seven graphs starts with the pentagon, corresponding to $(5,2,0,1)$. Then there are the Petersen graph with parameters $(10,3,0,1)$, the 
Clebsch graph with parameters $(16,5,0,2)$, the Hoffman-Singleton graph with parameters $(50,7,0,1)$, the Sims-Gewirtz graph with parameters $(56,10,0,2)$, the $M_{22}$ graph with $(77,16,0,4)$, and finally, the Higman-Sims graph with parameter set $(100,22,0,6)$. All these graphs are known to be unique for the corresponding parameter sets, see $[9,10,20]$ for more on these graphs.

It is generally believed that no further tfSRG exists. This is a very difficult open problem and nobody has a clear idea of a possible approach. Resolving the existence or uniqueness question for tfSRG even for one new feasible set of parameters is considered to be a major achievement. There are about 30 feasible arrays of tfSRGs till $v=2000$. Finally, one of the rare nonexistence result is about $(324,57,0,12)$, whose nonexistence was shown by Gavrilyuk and Makhnev, see [14].

Acknowledgements. The author acknowledges King Fahd University of Petroleum and Minerals for supporting this research.

\section{REFERENCES}

[1] Alfuraidan, M. R. Imprimitive distance-transitive graphs. Thesis (Ph.D.)-Michigan State University. ProQuest LLC, Ann Arbor, MI, 2004. 104 pp.

[2] Alfuraidan, M. R. Bipartite distance-transtive doubles with primitive halved of diameter two. Graphs and Combinatorics 29 (2013), no. 5, 1151-1174

[3] Alfuraidan, M. R. Antipodal distance-transtive covers with primitive quetient of diameter two. Discret. Math. 313 (2013), no. 21, 2409-2422

[4] Alfuraidan, M. R.; Hall, J. I.; Laradji, A. Antipodal distance-transtive covers of generalized polygons. J. Algebr. Comb. 48 (2018), no. 4, 607-626

[5] Alfuraidan, M. R.; Hall, J. I. Imprimitive distance-transtive graphs with primitive core of diameter at least 3. Michigan Math J. 58 (2009), 31-77

[6] Alfuraidan, M. R.; Hall, J. I. Smith's Theorem and a characterization of the 6-cube as distance-transtive graph. J. Algebraic Combin. 24 (2006), 195-207

[7] van Bon, J. T. M.; Brouwer, A. E. The distance-regular antipodal covers of classical distance-regular graphs. Combinatorics (Eger, 1987), 141-166, Colloq. Math. Soc. János Bolyai, 52, North-Holland, Amsterdam, 1988.

[8] Bannai, E. Maximal subgroups of law rank of finite symmetric and alternating groups. J. Fac. Sci. Univ. Tokyo Sect. I A 18 (1972), 475-486

[9] Brouwer, A. E.; Cohen, A. M.; Neumaier, A. Distance-regular Graphs, Springer, Berlin, 1989.

[10] Brouwer, A. E.; Van Maldeghem, H. Strongly regular graphs, Online, 2021.

[11] Buekenhout, F.; Van Maldeghem, H. A characterization of some rank 2 incidence Geometries by their automorphism group. Mitt. Math. Sem. Giessen no. 218 (1994).

[12] Foulser, D. A. Solvable primitive permutation groups of low rank. Trans. Amer. Math. Soc. 143 (1969), 1-54.

[13] Foulser, D. A.; Kallaher, M. J. Solvable, flag-transitive rank 3 collineation groups. Geom. Dedicata 7 (1978), 111-130.

[14] Gavrilyuk, A. L.; Makhnev, A. A. On Krein Graphs without Triangles. Doklady Mathematics 72 (2005), no. 1, 591-594.

[15] Hubaut, X. L. Strongly regular graphs. Discrete Math. 13 (1975), 357-381.

[16] Hemmeter, J. Halved graphs, Johnson and Hamming graphs. Utilitas Math. 25 (1984), 115-118.

[17] Hemmeter, J. Distance-regular graphs and halved graphs. Europ. J. Combin. 7 (1986), 119-129.

[18] Higman, D. G. Finite permutation groups of rank 3. Math. Z. 86 (1964), 145-156.

[19] Kantor, W. M.; Liebler, R. A. The rank 3 permutation representations of the finite classical groups. Trans. Amer. Math. Soc. 271 (1982), 1-71

[20] Klin, M.; Ziv-Av, M. Computer Algebra Investigation of Known Primitive Triangle-Free Strongly Regular Graph, Fifth International Symposium on Symbolic Computation in Software Science, Symposium Proceedings, Research Institute for Symbolic Computation, Johannes-Kepler University Linz, Austria, 2013.

[21] Liebeck, M. W. The affine permutation groups. Proc. Math Soc. 54 (1987), no. 3, 477-516.

[22] Liebeck, M. W.; Saxl, J. The finite primitive permutation groups of rank three. Bull. London Math. Soc. 18 (1986), 165-172.

[23] Liebeck, M. W.; Praeger, C. E.; Saxl, J. Distance-transitive graphs with symmetric or alternating automorphism group. Bull. Austral. Math. Soc. 35 (1987), 1-25

[24] Jurišić, A. Distance regular antipodal covers of strongly regular graphs, Master Eassay, University of Waterloo, April 1990. 
[25] Jurišić, A. Antipodal covers, Ph. D Thesis, University of Waterloo, January 1995.

[26] Muzychuk, M. A classification of one-dimensional affine rank three graphs. Discret. Math. 344 (2021), no. 7, 112400.

[27] Norton, S. P. On the group Fi $i_{24}$. Geom. Dedicata 25 (1988), 483-501.

[28] Praeger, C. E.; Saxl, J.; Yokoyama, K. Distance-transitive graphs and finite simple groups. Proc. London Math. Soc. 55 (1987), no. 3, 1-21.

[29] Smith, D. H. Primitive and imprimitive graphs. Quart. J. Math. Oxford 22 (1971), no. 2, 551-557.

[30] Soicher, L. H. Three new distance-regular graphs. Europ. J. Combin. 14 (1993), 501-505

[31] Skresanov, S. V. On 2-closures of rank 3 groups. ARS Mathematica Contemporanea 21 (2021), no. 1, \#P1.08

King Fahd University of Petroleum and Minerals

DEPARTMENT OF MATHEMATICS

P.O. Box 5046, DHAHRAN 31261, SAUdi ARABIA

Email address: montherakfupm. edu.sa

King Fahd University of Petroleum and Minerals

INTERDISCIPLINARY CENTER OF SMART MOBILITY AND LOGISTICS

P.O. Box 5067, DHAHRAN 31261, SAUdi ARABiA

Email address: monther@kfupm. edu.sa 